\title{
Asociación entre los factores sociodemográficos, obstétricos y patológicos con la muerte fetal tardía: estudio de casos y controles en un hospital de Perú
}

\section{Association between sociodemographic, obstetric and pathologic factors with late fetal death: a case-control study in a peruvian hospital}

\author{
Andrés Modesto Martínez-López ${ }^{1, a}$, Javier Roger Raúl Vargas-Herrera ${ }^{1, b}$, Elsy Haydee Mini-Díaz ${ }^{1, c}$ \\ ${ }^{1}$ Facultad de Medicina, Universidad Nacional Mayor de San Marcos. Lima, Perú. \\ ${ }^{a}$ Obstetra, magister en salud pública \\ ${ }^{\mathrm{b}}$ Médico cirujano, magister en epidemiologia. ORCID: https://orcid.org/0000-0002-1910-602X \\ ${ }^{\circ}$ Médico cirujano, magister en salud pública y gestión sanitaria. ORCID: https://orcid.org/0000-0002-7941-6708
}

An Fac med. 2019;80(3):322-6 / DOI: https://10.15381/anales.803.16855

\section{Correspondencia:}

Andrés Modesto Martínez-López

andresm_25@hotmail.com

Recibido: 18 de abril 2019

Aceptado: 15 de julio 2019

Publicación en línea: 30 de setiembre

2019

Conflictos de interés: Los autores

declaran no tener conflictos de interés.

Fuente de financiamiento:

Autofinanciado

Citar como: Martinez-López AM, VargasHerrera JR, Mini-Diaz EH. Asociación entre los factores sociodemográficos, obstétricos y patológicos con la muerte fetal tardía: estudio de casos y controles en un hospital de Perú. An Fac med. 2019;80(3):322-6.DOI: https://10.15381/ anales.803.16855

\section{Resumen}

Introducción: En el Perú, poco se conoce acerca de los factores de riesgo asociados a la muerte fetal tardia, lo que se considera información valiosa para implementar estrategias de prevención. Objetivos: Evaluar la asociación entre factores sociodemográficos, obstétricos y patológicos y la muerte fetal tardía en un hospital peruano. Métodos: Estudio retrospectivo de casos y controles entre el mes de julio de 2014 y junio de 2016. Los casos fueron gestantes que tuvieron muerte fetal intrauterina, y los controles fueron gestantes que tuvieron un recién nacido vivo. Se evaluaron factores como nivel educativo, ocupación, número de controles prenatales (CPN), y la presencia de complicaciones como parto pre-termino, pre-eclampsia o retardo del crecimiento intrauterino (RCIU) Resultados: Se incluyeron 60 casos y 120 controles. El nivel educativo primario (OR: 4,3; IC 95\% 1,0-18,0), la ocupación trabajadora (OR: 3,8; IC 95\% 1,3-11,0), no tener CPN (OR: 21,0; IC 95\% 2,6-170,1), la pre-eclampsia (OR: 4,1; IC 95\% 1,3-12,7), el parto pretérmino (OR: 10,1; IC 95\% 4.7-21,7) y RCIU (OR: 7,0; IC 95\% 2,4-20,5) se asociaron con la mortalidad fetal. Conclusión: Existen factores de riesgo sociodemográficos, obstétricos y patológicos asociados a tener muerte fetal tardía.

Palabras clave: Muerte Fetal; Factores de Riesgo; Atención Prenatal; Estudios Epidemiológicos (fuente: DeCS BIREME).

\section{Abstract}

Introduction: In Peru, a little is known about risk factors associated with late fetal death. This is a valuable information, which have to be used in order to implement preventional strategies. Objectives: To evaluate association between late fetal death and social, demographical, obstetrical and pathological factors in a peruvian hospital. Methods: A retrospective study of cases and controls between July 2014 and June 2016. The cases were pregnant women who had intrauterine fetal death and controls were pregnant women who had a newborn alive. Educational level, profession, number of prenatal care consults (CPN), and complications as preterm birth, pre-eclampsia or intrauterine growth retardation (RCIU) were evaluated as possible associated factors. Results: 60 cases and 120 controls were included. Primary education level $(\mathrm{OR}=4,3 ; 95 \% \mathrm{Cl}=$ 1,0-18,0), working occupation (OR $=3,8 ; 95 \% \mathrm{Cl}=1,3-11,0)$, no CPN $(\mathrm{OR}=21,0 ; 95 \% \mathrm{Cl}=2,6-170,1)$, pre-eclampsia $(\mathrm{OR}=4,1 ; 95 \% \mathrm{Cl}=1,3-12,7)$, preterm birth $(\mathrm{OR}=10,1 ; 95 \% \mathrm{Cl}=4,7-21,7)$ and $\mathrm{RCIU}(\mathrm{OR}$ $=7.0 ; 95 \% \mathrm{Cl}=2.4-20.5)$ were associated with fetal mortality. Conclusion: There are social, demographical, obstetrical and pathological risk factors associated with late fetal death.

Keywords: Fetal Death; Risk Factors; Prenatal Care; Epidemiological Studies (source: MeSH NLM). 


\section{INTRODUCCIÓN}

A nivel mundial ocurren aproximadamente 2,6 millones de muertes fetales cada año (fetos $>28$ semanas o $>1000 \mathrm{~g}$ de peso $)^{(1,2)}$. En Estados Unidos ocurren 7 muertes fetales tardías por cada 1000 recién nacidos vivos; mientras que en España la tasa de mortalidad fetal tardía anual entre 2000 y 2011 fue del 3-4 por 1000 nacidos vivos ${ }^{(3)}$. En el Perú, durante el año 2013 se determinó una tasa de muerte fetal tardía de 8,6 por mil embarazos ${ }^{(4)}$.

Debido a las diferentes definiciones de muerte fetal usadas en cada país, aproximadamente el $98 \%$ de muertes ocurren en los países en vías de desarrollo, cuyas tasas de mortalidad varían de 9 a 34 muertes fetales por cada 1000 nacidos vivos, comparado con 3,1 muertes fetales por cada 1000 nacidos vivos en los países desarrollados ${ }^{(1,2,5)}$.

Instituciones como la Organización Mundial de la Salud (OMS) recomienda el uso del término de mortalidad fetal tardía; mientras que la clasificación internacional estadística de enfermedades y problemas relacionados con la salud (CIE-10) define la mortalidad fetal tardía como la tasa de fetos nacidos muertos mayores de 28 semanas, mayores de $1000 \mathrm{~g}$ de peso, o con una longitud cráneo-rabadilla mayor de $35 \mathrm{~cm}^{(6)}$.

En las últimas décadas, el estudio de la mortalidad fetal tardía tiene mayor importancia debido a la identificación de la estrecha dependencia que guarda con los factores biológicos, demográficos y sociales, que hacen de ella un indicador sensible no sólo durante este suceso final, sino las repercusiones y la morbilidad que la preceden ${ }^{(7)}$. Adicionalmente, estudios previos han encontrado discrepancias en los registros de muerte materna y fetal tanto en Lima como el Callao ${ }^{(8)}$; y son poco concluyentes en lo relacionado a casos de muerte fetal tardia ${ }^{(9)}$.

Por ello, el objetivo de este estudio fue determinar los factores sociodemográficos y obstétricos asociados a la muerte fetal tardía en el Hospital Nacional Daniel Alcides Carrión (HNDAC), Callao, Perú.

\section{MÉTODOS}

\section{Diseño del estudio}

Se realizó un estudio retrospectivo, observacional y analítico de casos y con- troles en gestantes que fueron atendidas en el HNDAC del Ministerio de Salud, ubicado en el Callao, entre julio del 2014 y junio del 2016. Se incluyeron como casos a las gestantes mayores de 28 semanas que tuvieron una muerte fetal intrauterina. Como controles fueron seleccionadas las gestantes de 28 semanas o más, cuyo resultado del parto fue un recién nacido vivo. Los casos y controles fueron emparejados por fecha de atención del parto y edad de la madre en una proporción de 1:2. Para determinar el tamaño de la muestra, se utilizó la fórmula de casos y controles comparando proporciones. La prevalencia para los casos fue de $23 \%$ (p1), para la determinación de la prevalencia en los controles se aplicó la prevalencia de $6 \%$ con una confianza del 95\% (Z alfa) y un poder del 80\% (Z beta).

\section{Variables de estudio}

Se utilizó una ficha de recolección de datos validada por expertos para recolectar información sociodemográfica, obstétrica, patologías asociadas e información perinatal de las historias clínicas materno-perinatales. Los factores sociodemográficos incluyeron la edad, estado civil, nivel educativo, nivel económico, ocupación, consumo de tabaco y alcohol. Los factores obstétricos incluyeron, el control prenatal (CPN), paridad, muerte fetal previa, estado nutricional, hemorragia antes del parto, desprendimiento prematuro de placenta (DPP), placenta previa, circular de cordón y trauma materno. Los hallazgos patológicos de la madre incluyeron, la diabetes gestacional, preeclampsia, hipertensión crónica e infección del trato urinaria (ITU). La información perinatal incluyó la edad gestacional del producto, sexo fetal y retardo de crecimiento intrauterino (RCIU).

\section{Análisis estadístico}

Se realizaron pruebas bivariadas con el test de Chi cuadrado con un valor de $\mathrm{p}<0,05$ y se calcularon Odds ratio (OR) con intervalos de confianza (IC) del 95\% para determinar la asociación entre los factores sociodemográficos, obstétricos y patológicos con la muerte fetal tardía. Los análisis se realizaron en el software IBM SPSS Statistics for Windows, Versión 22.0 (IBM Corp. $2013^{\circledR}$ ).

\section{Aspectos éticos}

El estudio fue aprobado por el Comité de Ética del Hospital Nacional Daniel Alcides Carrión. Los investigadores mantuvieron absoluta confidencialidad de la información obtenida de las historias clínicas materno-perinatales.

\section{RESULTADOS}

Durante el periodo de estudio 9961 gestantes fueron atendidas en el HNDAC. De estas gestantes, 110 tuvieron una muerte fetal intrauterina (11 por mil nacidos vivos 110/9961). El cálculo del tamaño muestral fue determinado por la prevalencia de casos y controles, con una confianza del $95 \%$ y un poder del $80 \%$ aplicando la fórmula para estudios analíticos obteniéndose 60 casos y 120 controles en una proporción de 1:2.

\section{Factores sociodemográficos}

El promedio de edades fue similar entre los casos y controles (27,5 años vs. 26,9 años). El nivel educativo primario (OR: 4,3; IC 95\% 1,0-18,0; p<0,030) y la ocupación trabajadora dependiente (OR: 3,8; IC 95\% 1,3-11,0; $\mathrm{p}<0,010$ ) se asociaron con la muerte fetal tardía. Los otros factores sociodemográficos fueron similares en los casos y controles (Tabla 1).

\section{Factores obstétricos}

Las gestantes que tuvieron menos de cinco CPN (OR: 2,2; IC95\% 1,1-4,8; $\mathrm{p}<0,033$ ) o no tuvieron ningún CPN (OR: 21,0; IC 95\% 2,6-170,1; $p<0,0001$ ) tuvieron mayor riesgo de tener una muerte fetal tardía (Tabla 2).

\section{Hallazgos patológicos}

La pre-eclampsia (OR: 4,1; IC95\% 1,3-12,7; $p<0,011)$, el parto pretérmino (OR: 10,1; IC 95\%: 4,7-21,7; $p<0,0001$ ) $y$ el RCIU (OR: 7,0; IC 95\% 2,4-20,5; $\mathrm{p}<0,0001)$ se asociaron con la muerte fetal tardía (Tabla 3).

\section{DISCUSIÓN}

Los hallazgos encontrados en el presente estudio están relacionados con el bajo nivel educativo, la ausencia de con- 
Tabla 1. Factores sociodemográficos asociados con muerte fetal tardia en el Hospital Nacional Daniel Alcides Carrión, 2014-2016.

\begin{tabular}{|c|c|c|c|c|c|}
\hline \multirow{3}{*}{ Factor } & \multicolumn{4}{|c|}{$n(\%)$} & \multirow[b]{2}{*}{ Valor $p^{*}$} \\
\hline & Total & Casos & Controles & OR (IC 95\%) & \\
\hline & $n(\%)$ & $(n=60)$ & $(n=120)$ & & \\
\hline \multicolumn{6}{|l|}{ Edad materna (años) } \\
\hline $15-19$ & $29(16,1)$ & $9(15)$ & $20(16,7)$ & $0,9(0,4-2,1)$ & 0,774 \\
\hline $20-24$ & $41(22,8)$ & $15(25)$ & $26(21,7)$ & $1,2(0,6-2,5)$ & 0,615 \\
\hline $25-29$ & $47(26,1)$ & $13(21,7)$ & $34(28,3)$ & $0,7(0,3-1,5)$ & 0,337 \\
\hline $30-34$ & $37(20,6)$ & $13(21,7)$ & $24(20)$ & $1,1(0,5-2,4)$ & 0,794 \\
\hline $35-39$ & $16(8,9)$ & $6(10)$ & $10(8,3)$ & $1,2(0,4-3,5)$ & 0,711 \\
\hline $40-44$ & $9(5)$ & $3(6)$ & $6(5)$ & $1(0,2-4,1)$ & 1,000 \\
\hline $45-49$ & $1(0,6)$ & $1(1,7)$ & $0(0,0)$ & $0,9(0,9-1,0)$ & 0,156 \\
\hline \multicolumn{6}{|l|}{ Estado Civil } \\
\hline Soltero & $28(15,6)$ & $12(20)$ & $16(13,3)$ & $1,6(0,7-3,7)$ & 0,245 \\
\hline Conviviente & $129(71,7)$ & $39(65)$ & $90(75)$ & $0,6(0,3-1,2)$ & 0,160 \\
\hline Casado & $23(12,8)$ & $9(15)$ & $14(11,7)$ & $1,3(0,5-3,3)$ & 0,528 \\
\hline \multicolumn{6}{|l|}{ Nivel educativo } \\
\hline Primaria & $9(5)$ & $6(10)$ & $3(2,5)$ & $4,3(1,0-18,0)^{* *}$ & 0,030 \\
\hline Secundaria & $142(78,9)$ & $46(76,7)$ & $96(80)$ & $0,8(0,4-1,7)$ & 0,605 \\
\hline $\begin{array}{l}\text { Superior no } \\
\text { universitaria }\end{array}$ & $22(12,2)$ & $5(8,3)$ & $17(14,2)$ & $0,6(0,2-1,6)$ & 0,260 \\
\hline Superior universitaria & $7(3,9)$ & $3(5)$ & $4(3,3)$ & $1,5(0,3-7,1)$ & 0,586 \\
\hline \multicolumn{6}{|l|}{ Ocupación } \\
\hline Ama de casa & $134(74,4)$ & $42(70)$ & $92(76,7)$ & $0,7(0,4-1,4)$ & 0,334 \\
\hline Estudiante & $11(6,1)$ & $3(5)$ & $8(6,7)$ & $0,7(0,2-2,9)$ & 0,660 \\
\hline $\begin{array}{l}\text { Trabajadora } \\
\text { dependiente }\end{array}$ & $16(8,9)$ & $10(16,7)$ & $6(5)$ & $3,8(1,3-11,0)^{* *}$ & 0,010 \\
\hline Comerciante & $19(10,6)$ & $5(8,3)$ & $14(11,7)$ & $0,7(0,2-2,0)$ & 0,493 \\
\hline
\end{tabular}

OR, odds ratio; IC, intervalo de confianza.

*Chi cuadrado; **p<0,05

No se encontraron hallazgos de antecedente de consumo de tabaco, consumo de alcohol y antecedentes de muerte fetal tardía entre los casos y controles

trol prenatal y controles incompletos, así como casos asociados a factores patológicos como la preeclampsia y antecedentes de RCIU y parto pretérmino. Nuestros de Fustamante ${ }^{(10)}$ y Martínez Váldez ${ }^{(11)}$ quienes reportaron que las gestantes que tuvieron menor nivel educativo y que la- resultados son similares a los hallazgos

Tabla 2. Factores obstétricos asociados con muerte fetal tardía en el Hospital Nacional Daniel Alcides Carrión, 2014-2016.

\begin{tabular}{|c|c|c|c|c|c|}
\hline \multirow{3}{*}{ Factor } & \multirow{3}{*}{$\begin{array}{l}\text { Total } \\
\text { n (\%) }\end{array}$} & \multicolumn{2}{|c|}{ n (\%) } & \multirow{3}{*}{ OR (IC 95\%) } & \multirow{3}{*}{ Valor $p^{*}$} \\
\hline & & Casos & Controles & & \\
\hline & & $(n=60)$ & $(n=120)$ & & \\
\hline \multicolumn{6}{|l|}{ Control prenatal } \\
\hline Sin CPN & $11(6,1)$ & $9(15)$ & $2(1,7)$ & $21(2,6-170,1)^{* *}$ & 0,000 \\
\hline De 1 a 4 CPN & $35(19,4)$ & $17(28,3)$ & $18(15)$ & $2,2(1,1-4,8)^{*}$ & 0,033 \\
\hline De 5 a más CPN & $134(74,4)$ & $34(56,7)$ & $100(83,3)$ & $0,2(0,1-0,5)$ & 0,000 \\
\hline \multicolumn{6}{|l|}{ Paridad } \\
\hline Primera gestación & $51(28,3)$ & $19(31,7)$ & $32(26,7)$ & $1,3(0,6-2,5)$ & 0,483 \\
\hline Dos o más gestaciones & $129(71,7)$ & $41(68,3)$ & $88(73,3)$ & $0,8(0,4-1,5)$ & 0,483 \\
\hline \multicolumn{6}{|l|}{ Estado nutricional } \\
\hline Normal & $82(47,9)$ & $24(40,6)$ & $58(51,7)$ & $0,6(0,3-1,2)$ & 0,167 \\
\hline Bajo peso & $23(13,4)$ & $8(13,5)$ & $15(13,3)$ & $1,0(0,4-2,6)$ & 0,958 \\
\hline Sobrepeso & $29(16,9)$ & $10(16,9)$ & $19(16,9)$ & $1,0(0,4-2,3)$ & 0,998 \\
\hline Obesidad & $37(21,6)$ & $15(25,4)$ & $22(19,6)$ & $1,8(0,8-3,7)$ & 0,132 \\
\hline
\end{tabular}

CPN, control prenatal; OR, odds ratio; IC, intervalo de confianza.

*Chi cuadrado; $* * p<0,05$ boraban fuera de casa, tuvieron mayor riesgo de tener una muerte fetal tardía. En el presente estudio encontramos que las gestantes que tuvieron nivel educativo primario y ser trabajador dependiente durante el embarazo se asociaron con la muerte fetal tardía.

Adicionalmente, se encontró que el bajo nivel educativo de la madre fue factor de riesgo de muerte fetal. Postulamos que esta relación podría explicarse desde la condición socioeconómica, que al ser suficiente, provee mayor nivel educativo y con ello el acceso a los servicios de salud, así como periodos intergenésicos prolongados, situaciones que se conocen como factores que reducen el riesgo de mortalidad fetal y perinatal ${ }^{(12,13)}$.

En general, nuestros resultados son concordantes con lo reportado por la literatura internacional. De los factores socioeconómicos, el grado escolar bajo 
Tabla 3. Hallazgos patológicos asociados a muerte fetal tardía en el Hospital Nacional Daniel Alcides Carrión, 2014-2016. †

\begin{tabular}{lccccc}
\multirow{2}{*}{ Factor } & Total & Casos & Controles & OR (IC 95\%) & Valor $\mathbf{~}^{*}$ \\
\cline { 2 - 6 } ITU & $\mathbf{n}(\mathbf{\%})$ & $\mathbf{( n = 6 0 )}$ & $\mathbf{( n = 1 2 0 )}$ & & \\
\hline Pre-eclampsia & $38(21,1)$ & $12(20)$ & $26(21,7)$ & $0,9(0,4-1,9)$ & 0,796 \\
\hline Hemorragias & $14(7,8)$ & $9(15)$ & $5(4,2)$ & $4,1(1,3-12,7)^{* *}$ & 0,011 \\
\hline DPP & $6(3,3)$ & $6(10)$ & $0(0,0)$ & $0,9(0,8-1,0)$ & 0,000 \\
\hline Diabetes gestacional & $6(3,3)$ & $6(10)$ & $0(0,0)$ & $0,9(0,8-1,0)$ & 0,000 \\
\hline Hipertensión crónica & $3(1,7)$ & $3(5,0)$ & $0(0,0)$ & $1,0(0,9-1,0)$ & 0,014 \\
\hline Hallazgos del feto & $1(0,6)$ & $1(1,7)$ & $0(0,0)$ & $1,0(0,9-1,01)$ & 0,156 \\
\hline Parto a término & $134(74,4)$ & $27(45)$ & $107(89,2)$ & $0,1(0,05-0,2)$ & 0,000 \\
\hline Parto pretérmino & $46(25,6)$ & $33(55)$ & $13(10,8)$ & $10,1(4,7-21,7)^{* *}$ & 0,000 \\
\hline Sexo fetal masculino & $99(55,0)$ & $36(60)$ & $63(52,5)$ & $1,4(0,7-2,5)$ & 0,340 \\
\hline RCIU & $19(10,6)$ & $14(23,3)$ & $5(4,2)$ & $7,0(2,4-20,5)^{* *}$ & 0,000 \\
\hline
\end{tabular}

OR, odds ratio; IC, intervalo de confianza; DPP, desprendimiento prematuro de placenta; ITU, infección del tracto urinario; RCIU, retardo del crecimiento intrauterino.

No se encontraron hallazgos de placenta previa, circular de cordón, trauma materno y antecedentes de muerte fetal tardía entre los casos y controles

*Chi cuadrado; ${ }^{* *} p<0,05$

junto a un control prenatal deficiente, fueron los factores encontrados en la literatura ${ }^{(14,15)}$. Esto fue concluyente en nuestro estudio, el cual reportó que el perfil predominante en las mujeres con muerte fetal tardía atendidas en el Hospital Nacional Daniel Alcides Carrión fue el grado escolar bajo y ser trabajadora dependiente. En relación a los antecedentes que pueden mermar el desarrollo óptimo del producto, en nuestro estudio no encontramos antecedentes de tabaquismo y consumo de alcohol para el grupo de casos y controles.

En cuanto a los factores obstétricos, encontramos que las gestantes del HNDAC que tuvieron menos de 5 CPN o sin CPN, tuvieron altas posibilidades de tener una muerta fetal tardía ${ }^{(14,15)}$. Estos resultados son comparables con los hallazgos de Martínez y colaboradores quienes reportaron que los inadecuados CPN aumentan 9 veces el riesgo de presentar óbito ${ }^{(11)}$. Así, se recomienda sensibilizar a las mujeres en edad fértil sobre la importancia de los CPN, principalmente desde la etapa de preparación o preconcepción y también a las gestantes desde el inicio del primer CPN a fin de evitar muertes fetales.

Enfermedades como la hipertensión arterial sistémica, diabetes mellitus y obesidad son entidades patológicas consideradas epidemias del siglo XXI por su alta prevalencia a nivel nacional y com- plicaciones multiorgánicas. Estas patologías, al igual que las propias del embarazo como la diabetes gestacional y los estados hipertensivos del embarazo (preeclampsia leve y pre-eclampsia severa), se relacionan con múltiples eventos adversos maternos y fetales tales como la muerte intrauterina ${ }^{(12,16,17)}$. A igual que estudios previos ${ }^{(11,17)}$, encontramos que la pre-eclapmsia, el RCIU y el parto pretérmino se asociaron con la muerte fetal tardía. Esto podría explicarse por la ausencia de CPN, que tendría como consecuencia la identificación de estos factores en forma tardía.

En relación a las semanas de gestación, se encontró que la mayor frecuencia fue en pretermino con un 55\% (OR: 10,1); superior a lo reportado por Martínez ${ }^{(11)}$ quien reportó una frecuencia de 53\% (OR: 8,3) en este grupo de pacientes, llevando a identificar estos factores de forma tardía.

La restricción del crecimiento intrauterino es uno de los factores de riesgo de mayor relevancia en la morbimortalidad perinatal, según reportes de la literatura; así, se encuentra asociado con un aumento hasta 10 veces mayor de muerte fetal intrauterina en comparación a fetos $\sin \mathrm{RCIU}{ }^{(13,18)}$. En nuestro estudio, se evidenció que este factor estuvo asociado a un aumento hasta siete veces mayor de muerte fetal tardía.
En nuestro estudio se encontraron 9 pacientes con pre-eclampsia para el grupo de casos (15\%), de los cuales tres (30\%) cursaron con RCIU. Asimismo, del total de casos en los que se encontró RCIU (14 pacientes) sólo 7 (50\%) gestantes tuvieron 5 o más controles prenatales, lo que realza aún más la importancia de este factor. Adicionalmente, en el 33 $\%$ de casos de pre-eclampsia se encontró un desprendimiento prematuro de placenta. Estos resultados nos orientan a pensar que el entorno alrededor de estos casos es de poco conocimiento sobre el tema y poca investigación que brinde resultados que ayuden a incrementar estos conocimientos en el ámbito local. La óptima evaluación de la muerte fetal es tema de controversia y hay muy pocos datos que guíen a los clínicos ${ }^{(19)}$.

La causa más frecuente identificable de muerte fetal tardía en este estudio fue la "afección materna no especificada", reportada en 24 (40\%) pacientes. En segundo lugar, se encontraron 15 (25\%) casos con anencefalia, hidropesía, hidrocéfalo, malformaciones, 6 (10\%) casos con feto y recién nacido afectados por trastornos hipertensivos de la madre, 2 (3,3\%) casos con síndrome de recién nacido de madre con diabetes gestacional y $13(21,7 \%)$ casos con otras causas. Esta situación muestra que futuros estudios deberían ahondar en las dificultades de registro $y$, mediante seguimientos, explorar en qué casos suceden subregistros de causas de muerte fetal tardía.

La principal limitación de nuestro estudio radica en el diseño retrospectivo, ya que al obtener los datos de fuentes secundarias (historias clínicas), tuvieron que depurarse muchas historias clínicas para casos y controles por encontrarse incompletas o no contar con la historia clínica perinatal, ampliando el periodo de tiempo del estudio, y encontrar asociación entre los otros factores socioeconómicos, obstétricos y patológicos y la muerte fetal tardía. Sin embargo, para abordar esta limitación podría realizarse un estudio de cohorte en el futuro. Otra limitación de nuestro estudio es que no incluye resultados de autopsia, dificultando así el conocer las causas verdaderas de muerte fetal; si bien no era el objetivo de nuestra investigación, sería importante su identificación. 
Concluimos que en la población estudiada se encontró que las gestantes con menor nivel educativo, que trabajan de forma dependiente durante el embarazo, tienen menos de cinco CPN, preeclapmsia, un parto pretérmino y $\mathrm{RCIU}$, tienen un alto riesgo de tener una muerte fetal tardía. Por lo tanto, se siguiere vigilar e identificar estos factores de riesgo de forma oportuna, a fin de evitar la mortalidad fetal tardía.

\section{REFERENCIAS BIBLIOGRÁFICAS}

1. Lawn JE, Blencowe $H$, Pattinson R, Cousens $S$, Kumar R, Ibiebele I, et al. Stillbirths: Where? When? Why? How to make the data count? Lancet Lond Engl. 2011;377(9775):1448-63. DOI: 10.1016/ S0140-6736(10)62187-3

2. Cousens S, Blencowe H, Stanton C, Chou D, Ahmed S, Steinhardt L, et al. National, regional, and worldwide estimates of stillbirth rates in 2009 with trends since 1995: a systematic analysis. Lancet Lond Engl. 2011;377(9774):1319-30. DOI: 10.1016/ S0140-6736(10)62310-0

3. Valladares BZ, García DV, Buján CV, Couceiro NE, López RC. Muerte fetal intrauterina: ¿podemos actuar en su prevención? Rev Chil Obstet Ginecol. 2013;78(6):413-8. DOI: 10.4067/S071775262013000600002

4. Instituto Nacional de Estadistica e Informática (INEI). Encuesta Nacional de Demografía y Salud 2013 [On line]. INEI; 2013 [citado el 15 de abril 2019].
5. McClure EM, Wright LL, Goldenberg RL, Goudar SS, Parida SN, Jehan I, et al. The global network: a prospective study of stillbirths in developing countries. Am J Obstet Gynecol. 2007;197(3):247.e1-5. DOI: 10.1016/j.ajog.2007.07.004

6. Goldenberg RL, McClure EM, Saleem S, Reddy UM. Infection-related stillbirths. Lancet Lond Engl. 2010;375(9724):1482-90. DOI: 10.1016/S01406736(09)61712-8

7. Idrogo Tuesta SA. Control prenatal y su relación con la morbimortalidad perinatal en el Hospital Regional de Loreto durante el periodo de enero a diciembre del 2013 [Tesis para optar el Título Profesional de Médico-Cirujano]. Iquitos: Universidad Nacional de la Amazonia Peruana; 2014 [cité le 1 mai 2019]. Disponible en: http://repositorio.unapiquitos.edu. pe/handle/UNAP/3716

8. Espíritu N, Sacieta L, Pantoja L. Discrepancias en el registro de la mortalidad perinatal en Lima y Callao, según fuente de información. Rev Peru Med Exp Salud Publica. 2007;24(4):363-9.

9. Alejandro Choquihuayta K. Factores maternos asociados a muerte fetal tardía en el servicio de gineco-obstetricia del Hospital Carlos Lanfranco La Hoz en el año 2017 [Tesis para obtener el Título Profesional de Médico-Cirujano]. Lima: Universidad Privada San Juan Bautista; 2018 [citado 18 abri 2019]. Disponible en: http://repositorio.upsjb.edu. pe/handle/upsjb/1727

10. Fustamante-Núñez CF, Urquiaga-Sánchez T. Factores asociados a muerte fetal en dos hospitales referenciales de Lambayeque. Rev Cuerpo Méd HNAAA. 2017;10(4):192-6.

11. Martinez Valdés $C$. Epidemiologia de los factores de riesgo del Óbito Fetal, Hospital de Ginecología y Obstetricia Instituto Materno Infantil del Estado de México, 2012 [Tesis para obtener el diploma de especialista en Ginecología y Obstetricia]. Toluca: Universidad Autónoma del Estado de México; 2014 [citado 28 marzo 2019]. Disponible en: http://ri.uaemex. $\mathrm{mx} /$ handle/20.500.11799/14859

12. Silver RM, Varner MW, Reddy U, Goldenberg R, Pinar $\mathrm{H}$, Conway D, et al. Work-up of stillbirth: a review of the evidence. Am J Obstet Gynecol. 2007;196(5):433-44. DOI: 10.1016/j.ajog.2006.11.041

13. Druzin M, James S. Antepartum. Dans: Gabbe SG, Niebyl JR, Simpson JL, Landon MB, Galan HL, Jauniaux ERM, et al. Obstetrics: Normal and Problem Pregnancies, 7e. Edición: 7 éd. Philadelphia, PA: Elsevier; 2016.

14. Huertas Tacchino E. Epidemiologia de las muertes fetales ocurridas en el Instituto Especializado Materno Perinatal, 2003. Rev Peru Ginecol Obstet. 2007;53(3):199-202. DOI: 10.31403/rpgo.v53i1035

15. Panduro B JG, Pérez M JJ, Panduro M EG, Castro H $J F$, Vázquez G MD. Factores de riesgo prenatales en la muerte fetal tardia, Hospital Civil de Guadalajara, México. Rev Chil Obstet Ginecol. 2011;76(3):169-74. DOI: $10.4067 /$ S0717-75262011000300006

16. Dudley DJ. Diabetic-Associated Stillbirth: Incidence, Pathophysiology, and Prevention. Obstet Gynecol Clin. 2007;34(2):293-307. DOI: 10.1016/j.ogc.2007.03.001

17. Gabbe SG, Graves CR. Management of diabetes mellitus complicating pregnancy. Obstet Gynecol. 2003;102(4):857-68. DOI: 10.1016/j.obstetgynecol.2003.07.001

18. Schenone MH, Mari G. The MCA Doppler and its role in the evaluation of fetal anemia and fetal growth restriction. Clin Perinatol. 2011;38(1):83-102. DOI: 10.1016/j. clp.2010.12.003

19. M Kady S, Gardosi J. Perinatal mortality and fetal growth restriction. Best Pract Res Clin Obstet Gynaecol. 2004;18(3):397-410. DOI: 10.1016/j.bpobgyn.2004.02.009 